Planta (2000) 211: 370-375

\title{
The frequency of plasmodesmata increases early in the whole shoot apical meristem of Sinapis alba $\mathbf{L}$. during floral transition
}

\author{
Sandra Ormenese, Andrée Havelange, Roger Deltour, Georges Bernier \\ Laboratoires de Physiologie et de Morphologie, Département de Biologie végétale, Université de Liège, Sart-Tilman, 4000 Liège, Belgium
}

Received: 4 October 1999 / Accepted: 23 December 1999

\begin{abstract}
The frequency of plasmodesmata increases in the shoot apical meristem of plants of Sinapis alba L. induced to flower by exposure to a single long day. This increase is observed within all cell layers (L1, L2, L3) as well as at the interfaces between these layers, and it occurs in both the central and peripheral zones of the shoot apical meristem. The extra plasmodesmata are formed only transiently, from 28 to $48 \mathrm{~h}$ after the start of the long day, and acropetally since they are detectable in L3 $4 \mathrm{~h}$ before they are seen in L1 and L2. These observations indicate that (i) in the Sinapis shoot apical meristem at floral transition, there is an unfolding of a single field with increased plasmodesmatal connectivity, and (ii) this event is an early effect of the arrival at this meristem of the floral stimulus of leaf origin. Since (i) the wave of increased frequency of plasmodesmata is $12 \mathrm{~h}$ later than the wave of increased mitotic frequency (A. Jacqmard et al. 1998, Plant cell proliferation and its regulation in growth and development, pp. 67-78; Wiley), and (ii) the increase in frequency of plasmodesmata is observed in all cell walls, including in walls not deriving from recent divisions (periclinal walls separating the cell layers), it is concluded that the extra plasmodesmata seen at floral transition do not arise in the forming cell plate during mitosis and are thus of secondary origin.
\end{abstract}

Key words: Floral transition - Plasmodesmata formation - Secondary plasmodesmata - Shoot apical meristem - Sinapis (floral transition)

\section{Introduction}

The vegetative shoot apical meristem (SAM) in dicots has the shape of a dome and consists of a relatively small

\footnotetext{
Abbreviations: $\mathrm{CZ}=$ central zone; $\mathrm{LD}=$ long day; $\mathrm{Pd}=$ plasmodesmata; $\mathrm{PZ}=$ peripheral zone; $\mathrm{SAM}=$ shoot apical meristem; $\mathrm{SD}=$ short day

Correspondence to: Sandra Ormenese;

E-mail: sandra.ormenese@ulg.ac.be; Fax: + 32-4-3662960
}

number of undifferentiated dividing cells arranged in layers (Lyndon 1998). Cells of the two most superficial layers, L1 and L2, divide anticlinally (perpendicular to the meristem surface), except those L2 cells in the peripheral sectors involved in organogenesis that may divide periclinally (parallel to the meristem surface). Layers L1 and L2 collectively form the tunica which is responsible for surface growth of the SAM. The L3 cells, i.e. all cells below L1 and L2, divide in all planes and collectively form the corpus which is responsible for the growth in SAM volume. Given this pattern of orientation of cell divisions, cell walls in the SAM are not all of the same age: anticlinal walls in the tunica and all walls in the corpus are younger, i.e. derive from more recent divisions, than the periclinal walls in the tunica. A cytohistological zonation is usually superimposed on this layered structure, with the centrally located cells of both the tunica and corpus dividing less frequently and having less RNA and protein than the peripherally located cells of these tissues (Steeves and Sussex 1989; Lyndon 1998).

During the floral transition, although the layered structure persists, other aspects of SAM structure and activity are profoundly altered (Lyndon 1998). In particular, its cytohistological zonation fades away and ultimately disappears (Nougarède 1967; Lyndon 1998), and the pattern of gene activity is completely changed. For instance, up-regulation of some genes like Arabidopsis TFL1 or Sinapis SaMADS A and FPF1 is observed at particular times and in particular areas of the transitional SAM (Menzel et al. 1996; Bradley et al. 1997; Kania et al. 1997). This step-wise and tissuespecific transformation of the SAM points to a complex coordination network of cell activities (Huala and Sussex 1993; van der Schoot and Rinne 1999). Such a coordination demands proper communication channels between cells. Plasmodesmata $(\mathrm{Pd})$ provide such channels (Robards and Lucas 1990; Lucas et al. 1993) and it is thus quite possible that their frequency pattern and/or permeability may change at the floral transition. Studies on Pd at this developmental step are, however, very few (Goodwin and Lyndon 1983; Bergmans et al. 1993, 1997; Milyaeva and Nikiforova 1995, 1996) and so far 
have examined in each case the situation only at a single time point of the floral transition, possibly missing changes occurring at other times.

In the present work, our aim is to search for temporal and spatial changes in Pd frequency in ultrathin SAM sections using an experimental system in which the floral transition is easily controlled and of short duration. The experimental system used was the long-day (LD) plant Sinapis alba, a member of the Brassicaceae, which can be induced to flower by exposure to a single LD. In response to this $\mathrm{LD}$, a floral stimulus of leaf origin reaches the SAM at 16-28 $\mathrm{h}$ after the start of the LD and causes the transformation of the vegetative SAM into an inflorescence meristem (Bernier 1989). This meristem starts to initiate the first floral meristems of the terminal raceme roughly $2 \mathrm{~d}$ after arrival of the floral stimulus at the apex (Bernier 1989). Another advantage of this experimental system is that the changes affecting the cell division process within the SAM during these $2 \mathrm{~d}$ have been precisely described (Gonthier et al. 1987; Jacqmard et al. 1998). This is particularly important given that the 'primary' $\mathrm{Pd}$ are formed during cytokinesis (Robards and Lucas 1990; Lucas et al. 1993). These Pd are unbranched and present mainly in walls separating adjacent cells of a lineage. On the other hand, 'secondary' Pd are formed post-cytokinetically across all kinds of pre-existing walls in the SAM (Bergmans et al. 1997). These Pd may thus be present not only in walls separating cells of a lineage but mainly in walls separating cells belonging to two adjacent lineages. Secondary Pd may be unbranched, and then indistinguishable from primary Pd. Alternatively, they may be branched due to the fusion of neighbouring unbranched $\mathrm{Pd}$ or to the addition of new cytoplasmic bridges to an unbranched Pd. More details about the structure and function of primary and secondary Pd can be found in Kollmann and Glockmann (1999).

\section{Materials and methods}

Plant material. Plants of Sinapis alba L. (Etablissement Jardins, Nancy, France) were kept vegetative by growing them under 8-h short days (SDs), as described by Lejeune et al. (1988). When 2 months old, they were induced to flower by a single 22-h LD and then returned to the 8-h SD regime. These plants will be referred to as LD-induced in contrast to SD-control plants kept continuously under standard SD conditions. All light periods were provided with V.H.O. Sylvania fluorescent white tubes at an irradiance of $150 \mu \mathrm{mol}$ photons $\mathrm{m}^{-2} \mathrm{~s}^{-1}$ at the top of the plants.

Transmission electron microscopy. Ten rapidly defoliated SAMs of both SD-control and LD-induced plants were collected at different time intervals after start of the LD (28, 32, 36 and 48 h). Five different kinds of fixation were tested: (i) the classical double fixation with glutaraldehyde and osmium tetroxide (Ledbetter and Porter 1963), (ii) the same fixation plus tannic acid (Olesen 1979), (iii) osmium tetroxide-potassium ferricyanide fixation (Hepler 1981), (iv) double fixation with formaldehyde-calcium followed by potassium dichromate (Glauert 1965), and (v) potassium permanganate fixation (Luft 1956). Since our aim was not to describe the ultrastructure of the Pd but only to count them unequivocally, we chose the last fixation method which produced high-contrast membranes (as the appressed ER passed through the
Pd), and was rapid and easily utilized. Consequently, the collected samples were fixed in $2 \%$ potassium permanganate in $0.2 \mathrm{M}$ sodium cacodylate buffer $(\mathrm{pH} 7.4)$ for $45 \mathrm{~min}$ at $4{ }^{\circ} \mathrm{C}$. After dehydration in a graded alcohol series, the meristems were embedded in Epon. The experiment and SAM collection were done twice at a 1-year interval. Five to eight SAMs from the two experiments were randomly chosen for each collection time. Ultrathin longitudinal axial sections (approximately $80-90 \mathrm{~nm}$, pale silver interference color) were made in each apex with a Sorvall MT-2 ultra-microtome. In addition, ultrathin serial sections were made in some apices in order to reconstitute the three-dimensional structure of the obliquely sectioned $\mathrm{Pd}$. All the sections were collected on one-slot copper grids coated with $0.2 \%$ formvar in chloroform, and subsequently contrasted for $3 \mathrm{~min}$ with $4.6 \%$ uranyl acetate solution in $50 \%$ ethanol. The sections were finally examined with a Zeiss EM 900 electron microscope at $80 \mathrm{kV}$.

Determination of Pd frequency. Twenty non-contiguous cells from each SAM were selected in the three cell layers: six cells in the first layer (L1), six cells in the second layer (L2) and eight cells in the third layer (L3), taking care to exclude from L3 the flattening cells belonging to the pith-rib meristem (Fig. 1). The Pd were counted along the periclinal and anticlinal walls of these 20 selected cells at a magnification of $\times 20000$. We encountered linear single-stranded Pd (referred to as "unbranched" Pd) and Pd showing multicytoplasmic strands (referred to as "branched" Pd). In both cases, the entire structures (single unbranched or entire branched structures) were counted. The lengths of the periclinal and anticlinal walls of each selected cell were measured using image measurement software (SigmaScan Pro/Image; Jandel Scientific) on electron micrographs taken with a video editor and capture program (Video Studio 2; Ulead Systems) at a final magnification of $\times 20000$. The $\mathrm{Pd}$ frequency in a particular wall was expressed as the Pd number per $\mu \mathrm{m}$ of that wall. Values obtained for anticlinal and periclinal walls in each of the three cell layers were averaged for each collection time of the two experiments. Total numbers of cells examined per collection time varied between 100 and 160 .

Frequencies of Pd were also determined in the central $(\mathrm{CZ})$ and peripheral zones (PZ) of the SAM. In the ultrathin longitudinal axial sections, the whole SAM including L1, L2 and L3 cells was arbitrarily divided into three parts: the internal part representing the $\mathrm{CZ}$ and the two external parts together representing the PZ (Fig. 1). In both zones of each SAM, three cells were selected in each layer and examined for Pd frequency as explained above. This kind of analysis was performed only for SAMs collected $36 \mathrm{~h}$ after the start of the LD. Five SAMs from SD-control plants and five from LD-induced plants collected in two independent experiments (see above) were used. The total number of cells examined was 45

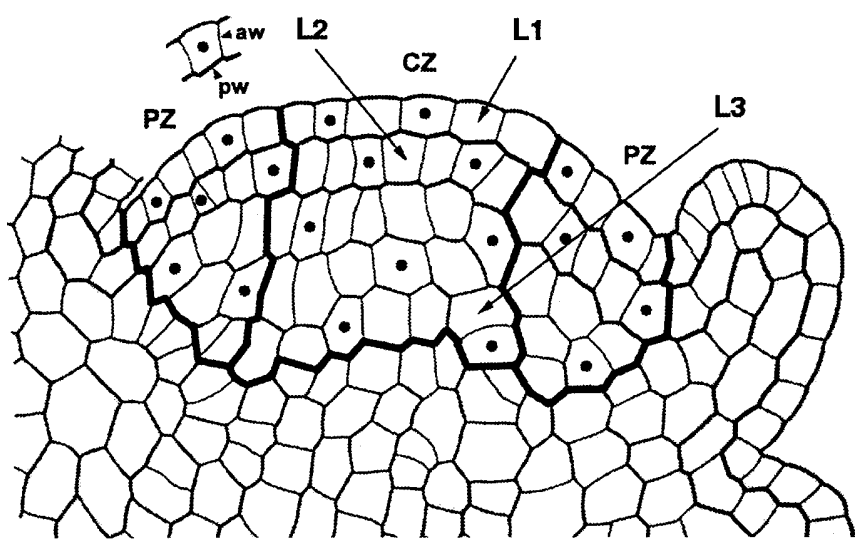

Fig. 1. Diagram showing how the selection of 20 non-contiguous cells (dotted cells) in the three cell layers (L1, L2 and L3) of each SAM was made. Thick lines indicate the borders of the central $(C Z)$ and peripheral $(P Z)$ zones. $a w$, anticlinal wall; $p w$, periclinal wall 
Table 1. Percentages of different types of Pd observed in the SAM of SD-control and LD-induced Sinapis alba plants. All SAMs from all collection times were pooled

\begin{tabular}{lllll}
\hline Treatment & \multicolumn{2}{l}{ Unbranched } & \multirow{2}{*}{ Branched } \\
\cline { 2 - 4 } & Complete & $\begin{array}{l}\text { Obliquely } \\
\text { sectioned Pd }\end{array}$ & Total & \\
\hline SD-control & 56 & 37 & 93 & 7 \\
LD-induced & 64 & 32 & 96 & 4 \\
\hline
\end{tabular}

for either the $\mathrm{CZ}$ or the PZ. The cells selected here were not the same as those used for the study of individual cell layers above.

Averages for SD-control and LD-induced plants were compared at each collection time using the Student's $t$-test at the $5 \%$ level of significance. In the rare cases of non-normal populations or populations with unequal variances, a Mann-Whitney Rank Sum test was used at the same level of significance. To compare the values obtained for SD-control plants at the different collection times, a one-way analysis of variance test (Anova) was used. All the statistics were performed using the statistics software SigmaStat (Jandel Scientific).

\section{Results}

The Pd were observed in all walls of all cells of the Sinapis SAM. Among the $10000 \mathrm{Pd}$ observed in this study, in both SD-control and LD-induced SAMs, about $95 \%$ of them appeared unbranched (Table 1), were present singly (Fig. 2A) or in groups of 2-10 (Fig. 2B). There was no structural difference between the
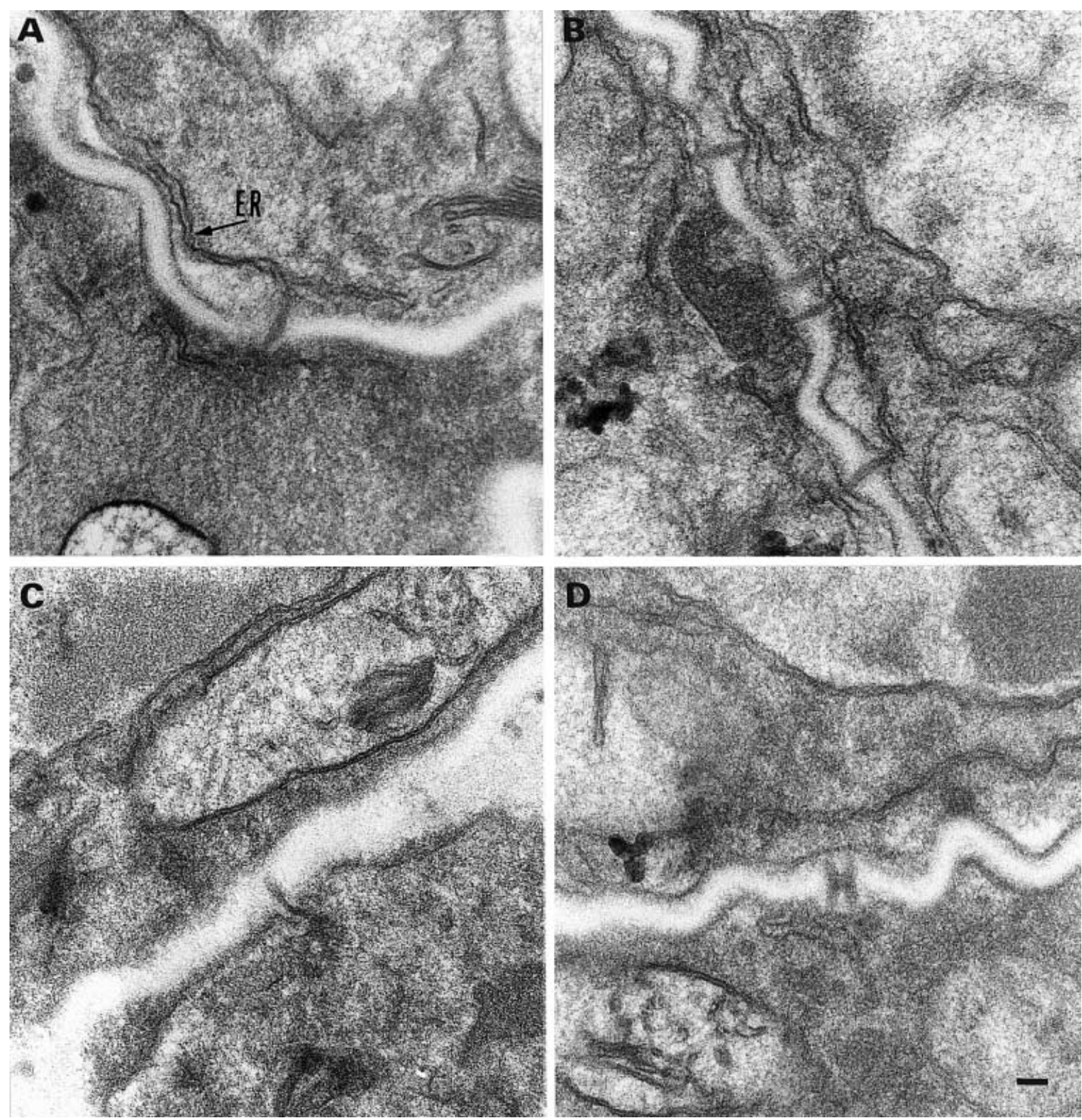

Fig. 2A-D. Ultrathin sections of the SAM of Sinapis alba. A An unbranched, complete, singly present Pd. B A group of unbranched Pd. C An obliquely sectioned unbranched Pd. D A branched Pd with a characteristic H-shaped structure. ER, endoplasmic reticulum. Bar $=0.1 \mu \mathrm{m}$ 


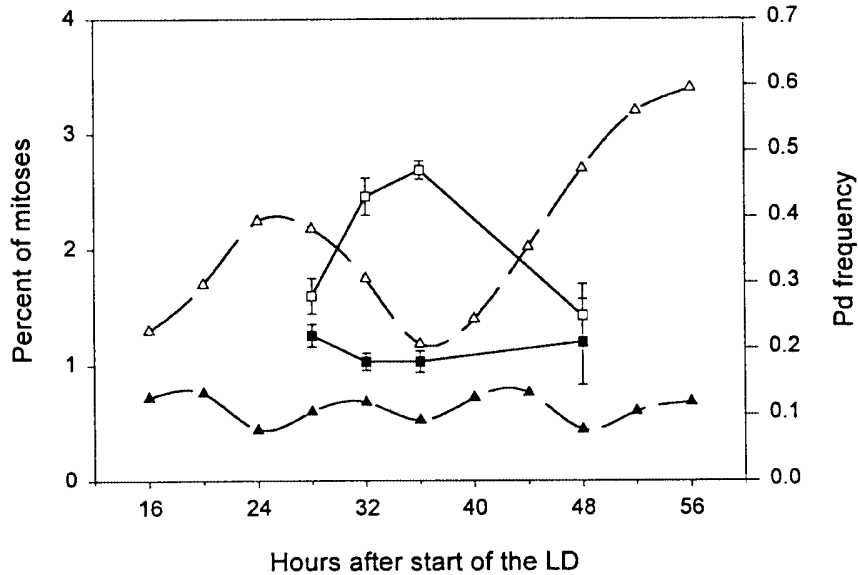

Fig. 3. Frequencies of Pd (number of Pd per $\mu \mathrm{m}$ wall length) $\pm \mathrm{SE}$ $(\boldsymbol{\square} ; \square)$ and mitotic indices $(\boldsymbol{\Delta} ; \triangle)$ in the whole SAM of SD-control (closed symbols) and LD-induced (open symbols) $S$. alba plants collected at various times after the start of inductive LDs. Data for mitotic indices are adapted from Jacqmard et al. (1998)

unbranched Pd observed in the different locations within the SAM. A majority of the unbranched Pd (about $60 \%$ ) were complete, i.e. had a distinct connection with the protoplasts of two adjacent cells, whereas the remainder of these Pd only had a distinct connection with the protoplast of one of two adjacent cells (Table 1, Fig. 2C). Serial sections showed that these Pd were in fact obliquely sectioned complete Pd. Occasionally, branched $\mathrm{Pd}$ were also observed (about $5 \%$ of the counted Pd) in both control and induced SAMs (Table 1, Fig. 2D). The branched Pd were all structurally similar, regardless of their position within the SAM.

Data concerning the frequencies of complete unbranched Pd for the whole SAM (all layers and zones included) are shown in Fig. 3. As indicated by the one- way analysis of variance, there were no statistically significant differences between the Pd frequencies in the SAM of SD-control plants at the different collection times. This frequency oscillated around $0.2 \mathrm{Pd}$ per $\mu \mathrm{m}$ of wall. Frequencies in the SAM of LD-induced plants were significantly increased at 28,32 and $36 \mathrm{~h}$ after the start of the LD. A frequency peak, corresponding to a 2.5 -fold increase, was observed at $36 \mathrm{~h}$.

Frequencies of complete unbranched Pd inside and between SAM layers can be found in Fig. 4. Twentyeight hours after the start of the LD, a small but significant increase of Pd frequency was observed inside the L3 layer of LD-induced SAMs compared with SDcontrol SAMs (Fig. 4A). There was, however, no statistically significant difference, using the Student's $t$-test, elsewhere between SAMs of control and induced plants. Thirty-two hours after the start of the LD, the Pd frequencies were markedly and significantly increased in the whole SAM of induced plants, i.e. inside L1, L2 and L3 and at the interfaces between these layers (Fig. 4B). A peak of Pd frequency was reached at $36 \mathrm{~h}$ after the start of the LD (Fig. 4C). At this stage, all Pd frequencies in the induced SAMs were about 2- to 4-fold higher than in control SAMs. At $48 \mathrm{~h}$ after the start of the LD, the Pd frequencies within the induced SAMs strongly decreased and returned to values that were not significantly different, using the Student's $t$-test, from control SAMs (Fig. 4D).

At the peak time, i.e. $36 \mathrm{~h}$ after the start of the LD, when the Pd frequencies were determined separately in the $\mathrm{CZ}$ and PZ of the SAM, a significant increase, in fact a doubling, of frequency was found in both zones (Fig. 5).

Similar conclusions were reached when, instead of considering the complete unbranched Pd only, all unbranched $\mathrm{Pd}$ (complete and obliquely sectioned $\mathrm{Pd}$ )
A $\quad 28 \mathrm{~h}$ after start of the LD

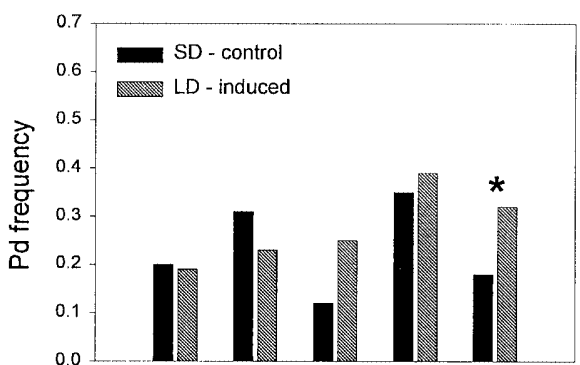

C $\quad 36 \mathrm{~h}$ after start of the LD



B $\quad 32 \mathrm{~h}$ after start of the LD

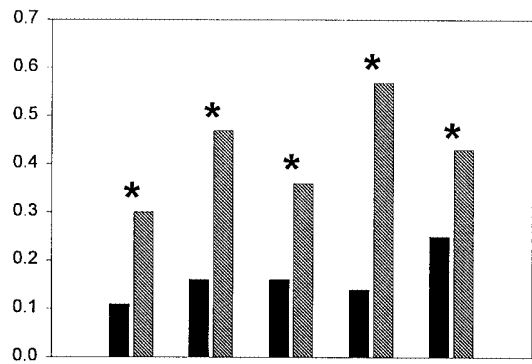

D $\quad 48 \mathrm{~h}$ after start of the LD

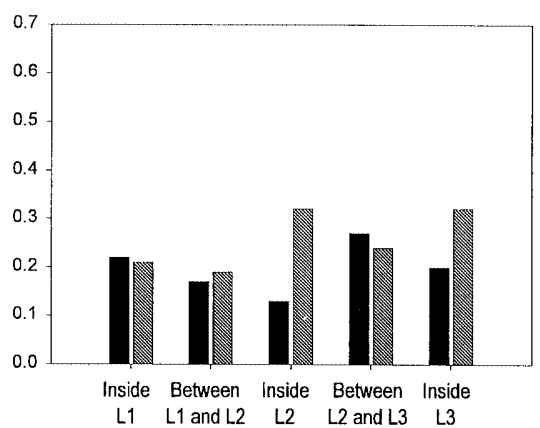

Fig. 4A-D. Frequencies of Pd (number of Pd per $\mu \mathrm{m}$ wall length) inside the L1, L2 and L3 and at the interfaces between these cell layers in the SAM of SD-control and LD-induced $S$. alba plants. Plasmodesmatal


$36 \mathrm{~h}(\mathbf{C})$, and $48 \mathrm{~h}(\mathbf{D})$ after the start of the LD. Data are for unbranched complete Pd. Asterisks indicate when values for LDinduced SAMs are significantly different from SD-control SAMs using the Student's $t$-test 


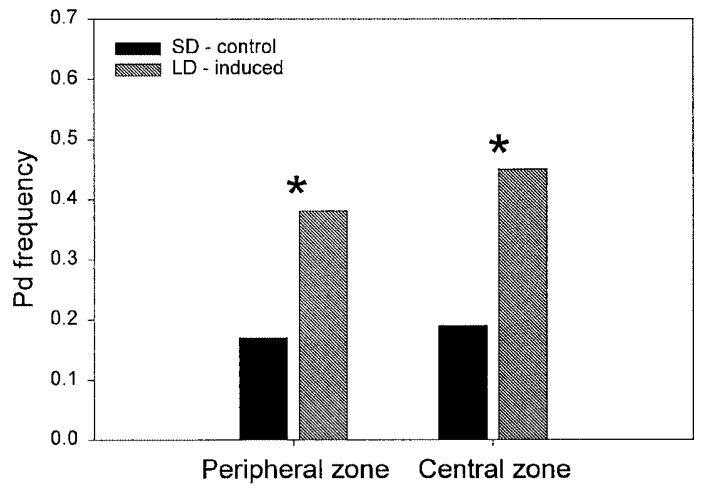

Fig. 5. Frequencies of $\mathrm{Pd}$ (number of $\mathrm{Pd}$ per $\mu \mathrm{m}$ wall length) inside the peripheral and central zones of the SAM of SD-control and LDinduced $S$. alba plants collected $36 \mathrm{~h}$ after the start of the LD. Data are for unbranched complete Pd. Asterisks indicate when values for LD-induced SAMs are significantly different from SD-control SAMs using the Student's $t$-test

and branched Pd were included in the calculations (data not shown).

\section{Discussion}

Our observations show that, during the floral transition of the SAM of Sinapis alba, the Pd frequency increases dramatically in the whole meristem, across its cell layers and cytohistological zones. This indicates that intercellular communications in the SAM are increased at this developmental switch. Since this change is ubiquitous, it may reflect the unfolding within the SAM at floral transition of a single field with increased Pd connectivity. Increased $\mathrm{Pd}$ connectivity may be related to increased cell homogeneity, corresponding possibly to the so-called 'prefloral phase' during which the zonation fades away in transitional SAMs, including the Sinapis SAM (Nougarède 1967; Bernier et al. 1981b; Lyndon 1998). A similar increase in Pd frequency was reported for the CZ of the transitional SAM of Rudbeckia bicolor, an LD plant of the family Asteraceae (Milyaeva and Nikiforova 1995, 1996). Although the Rudbeckia SAM was examined at a single time point of the floral transition ( $8 \mathrm{~d}$ after the start of the LD induction; at a stage corresponding apparently to the prefloral phase; Milyaeva et al. 1991), the PZ was not studied in Rudbeckia, and the structural details of the floral transformation of the SAM are very different in Asteraceae and Brassicaceae (Nougarède 1967; Bernier et al. 1981b). However, it is noteworthy that the Pd frequency changes were in the same direction in Rudbeckia and Sinapis.

Considering that primary $\mathrm{Pd}$ are formed at cytokinesis and cell divisions are commonly stimulated in SAMs at the floral transition (Bernier et al. 1981b), it is important to compare the kinetics of changes in $\mathrm{Pd}$ frequency with those in mitotic frequency (mitotic index) in our experimental system. As described by Jacqmard et al. (1998), in the SAM of Sinapis plants induced by one $22-\mathrm{h} \mathrm{LD}$, there is an early increase of the mitotic index which is first detectable at $16 \mathrm{~h}$ after the start of the LD, peaking at 24-28 h, and subsiding until $36 \mathrm{~h}$. A second wave of mitoses then starts at $40 \mathrm{~h}$ and peaks $20 \mathrm{~h}$ later, just a few hours before the initiation of the floral meristem by the SAM. Since its discovery (Bernier et al. 1967), early mitotic activation has always been found to occur with the same timing and amplitude; therefore, the data of Jacqmard et al. (1998) have been included in Fig. 3. The first wave of mitoses (from 16 to $36 \mathrm{~h}$ ) is about $12 \mathrm{~h}$ in advance of the wave of increased Pd frequency (Fig. 3) and, thus Pd formation does not relate to cytokinesis but rather to the subsequent processes of cell enlargement and wall stretching. This conclusion is further supported by the fact that $\mathrm{Pd}$ frequency returns to basal control levels found at $48 \mathrm{~h}$, i.e. during the second mitotic wave (Fig. 3). Given that the duration of cytokinesis is usually very short (Alberts et al. 1994), these observations suggest that most, if not all, of the supplementary Pd seen at the floral transition are not formed in developing cell plates, but instead in already existing walls. This implies that these newly formed Pd are secondary in nature.

This suggestion is reinforced by the further observation that $\mathrm{Pd}$ frequencies increase not only in the relatively young walls of the transitional SAM (anticlinal walls of L1 and L2 and all walls of L3) but also in its old walls (periclinal walls between L1 and L2 and between L2 and L3). A survey made on 60 transitional SAMs of Sinapis, collected during the experimental period covered in our work, has confirmed that the mitotic figures observed in L1 and L2 are all anticlinal (except in the L2 area involved in the initiation of the last leaves; data not shown). Thus, the supplementary $\mathrm{Pd}$ seen in the periclinal walls of L1 and L2 are necessarily of secondary origin. Collectively, our observations indicate that the supplementary Pd in all wall types of the transitional SAM of Sinapis are formed post-cytokinetically, and thus are of secondary origin.

Interestingly, these extra $\mathrm{Pd}$ are formed only transiently, since they are detectable during less than $1 \mathrm{~d}$, and acropetally, since they are detectable in L3 at $28 \mathrm{~h}$ and in L1 and L2 at $32 \mathrm{~h}$ only. These observations are consistent with the fact that, in plants caused to flower by a single favourable photoperiodic cycle, the export from leaves of the floral stimulus is transient and the movement of this stimulus towards the SAM is necessarily acropetal (Bernier et al. 1981a). The floral stimulus in Sinapis plants induced by one LD is known to move from leaves to SAM between 16 and $28 \mathrm{~h}$ following start of the LD (Bernier 1989). Being first detectable at $28 \mathrm{~h}$, the formation of extra Pd appears to be an early effect of the arrival of the floral stimulus, or one of its components, at the SAM.

The transient nature of the increase in Pd frequency can be explained by a transient activation of the process of secondary $\mathrm{Pd}$ formation followed by a dilution of these $\mathrm{Pd}$ due to increased mitotic frequency (second mitotic wave starting after $40 \mathrm{~h}$ ) and cell expansion (Havelange and Bernier 1974). In addition to this temporary biogenesis of extra secondary $\mathrm{Pd}$ in the Sinapis SAM at the floral transition, symplasmic 
regulation might involve alteration of the plasmodesmatal size-exclusion limit or of the compartmentalization of the SAM. Further work in Sinapis will attempt to determine whether these types of symplasmic alterations may occur at the transition to flowering and in which time-frame.

We are indebted to Dr. R.F. Lyndon (University of Edinburgh, UK) for critical reading of the manuscript and to Dr. C. van der Schoot (ATO-DLO, Wageningen, The Netherlands) for helpful discussion. This work was supported by grants from the Interuniversity Poles of Attraction Programme (Belgian State, Prime Minister's Office - Federal Office for Scientific, Technical and Cultural Affairs; P4/15) and the University of Liège (Fonds Spéciaux). Sandra Ormenese is grateful to F.R.I.A. for the award of a research fellowship. We thank Pierre Parkinson for photographic work, Sylvie Jacques for technical assistance, and Pierre Tocquin for the use of his computer facilities.

\section{References}

Alberts B, Bray D, Lewis J, Raff M, Roberts K, Watson JD (1994) Molecular biology of the cell, 3rd edn. Garland Publ, New York

Bergmans ACJ, de Boer D, van Bel AJE, van der Schoot C (1993) The initiation and development of Iris flowers - permeability changes in the apex symplasm. Flowering Newslett 16: 19-25

Bergmans ACJ, de Boer D, Derksen JWM, van der Schoot C (1997) The symplasmic coupling of L2-cells diminishes in early floral development. Planta 203: 245-252

Bernier G (1989) Events of the floral transition of meristems. In: Lord E, Bernier $G$ (eds) Plant reproduction: from floral induction to pollination. American Society of Plant Physiologists Series, vol I, Rockville, Maryland, pp 42-50

Bernier G, Kinet J-M, Bronchart R (1967) Cellular events at the meristem during floral induction in Sinapis alba L. Physiol Vég 5: $311-324$

Bernier G, Kinet J-M, Sachs RM (1981a) The physiology of flowering, vol I. CRC Press, Boca Raton, Florida, pp 83-103

Bernier G, Kinet J-M, Sachs RM (1981b) The physiology of flowering, vol II. CRC Press, Boca Raton, Florida, pp 35-53

Bradley D, Ratcliffe O, Vincent C, Carpenter R, Coen E (1997) Inflorescence commitment and architecture in Arabidopsis. Science 275: 80-83

Glauert AM (1965) Fixation and embedding of biological specimens. In: Kay DH (ed) Techniques for electron microscopy. Blackwell Sci Publ, Oxford, pp 166-212

Gonthier R, Jacqmard A, Bernier G (1987) Changes in cell-cycle duration and growth fraction in the shoot meristem of Sinapis during floral transition. Planta 170: 55-59

Goodwin PB, Lyndon RF (1983) Synchronisation of cell division during transition to flowering in Silene apices not due to increased symplast permeability. Protoplasma 116: 219-222
Havelange A, Bernier G (1974) Descriptive and quantitative study of ultrastructural changes in the apical meristem of mustard in transition to flowering. I. The cell and nucleus. J Cell Sci 15: 633-644

Hepler PK (1981) The structure of the endoplasmic reticulum revealed by osmium tetroxide-potassium ferricyanide staining. Eur J Cell Biol 26: 102-110

Huala E, Sussex IM (1993) Determination and cell interactions in reproductive meristems. Plant Cell 5: 1157-1165

Jacqmard A, Houssa C, Bernier G (1998) Control of the cell division cycle changes in the shoot meristem of Sinapis alba during the transition to flowering. In: Bryant JA, Chiatante D (eds) Plant cell proliferation and its regulation in growth and development. Wiley, New York, pp 67-78

Kania T, Russenberger D, Peng S, Apel K, Melzer S (1997) FPF1 promotes flowering in Arabidopsis. Plant Cell 9: 1327-1338

Kollmann R, Glockmann C (1999) Multimorphology and nomenclature of plasmodesmata in higher plants. In: van Bel AJE, van Kesteren WJP (eds) Plasmodesmata. Structure, function, role in cell communication. Springer, Berlin, pp 149-172

Ledbetter MC, Porter KR (1963) A 'microtubule' in plant cell fine structure. J Cell Biol 19: 239-250

Lejeune P, Kinet J-M, Bernier G (1988) Cytokinin fluxes during floral induction in the long day plant Sinapis alba L. Plant Physiol 86: 1095-1098

Lucas WJ, Ding B, van der Schoot C (1993) Plasmodesmata and the supracellular nature of plants. New Phytol 125: 435-476

Luft JH (1956) Permanganate - a new fixative for electron microscopy. J Biophys Biochem Cytol 2: 799-801

Lyndon RF (1998) The shoot apical meristem: its growth and development. Cambridge Univ Press, Cambridge

Menzel G, Apel K, Melzer S (1996) Identification of two MADS box genes that are expressed in the apical meristem of the longday plant Sinapis alba in transition to flowering. Plant J 9: 399408

Milyaeva EL, Golyanovskaya SA, Aksenova NP, Chailakhyan MKh (1991) Changes of protein composition in leaves and stem apices of bicolored coneflower with transition to flowering. Dokl Akad Nauk SSSR 316: 11-15

Milyaeva EL, Nikiforova VJ (1995) Symplastic connections in the stem apex of coneflower plants during floral transition. Russ $\mathbf{J}$ Plant Physiol 42: 640-645

Milyaeva EL, Nikiforova VJ (1996) Informational signaling in stem apical meristems during transition to flowering. Flowering Newslett 22: 26-33

Nougarède A (1967) Experimental cytology of the shoot apical cells during vegetative growth and flowering. Int Rev Cytol 21: 203251

Olesen P (1979) The neck constriction in plasmodesmata. Planta 144: $349-358$

Robards AW, Lucas WJ (1990) Plasmodesmata. Annu Rev Plant Physiol Plant Mol Biol 41: 369-419

Steeves TA, Sussex IM (1989) Patterns in plant development, 2nd edn. Cambridge Univ Press, Cambridge

van der Schoot C, Rinne PLH (1999) Networks for shoot design. Trends Plant Sci 4: 31-37 